\title{
Editorial
}

\section{Contribution of spectroscopic magnetic resonance imaging to target volume delineation in Gamma Knife Radiosurgery: Myth or reality?}

\author{
Dr. Fahd Derkaoui Hassani \\ Department of Neurosurgery, Cheikh Zaid International University Hospital, Rabat, Morocco. \\ Abulcasis International University of Health Sciences, Rabat, Morocco. \\ Mohammed V University of Rabat, Rabat, Morocco.
}

Brain malignancies are still associated with poor prognosis despite multimodal radiosurgical therapeutic approach using Gamma Knife (GK), CyberKnife (CK), and linear accelerator-based technologies [1]. These advances have significantly improved the treatment outcome. However, the surgical and radiosurgical concept is still "image-guided", and the success is closely related to precise tumor volume definition. The gross tumor volume (GTV) is defined as the visible contrast- enhancing lesion on magnetic resonance (MR) images with high three-dimensional spatial accuracy. Target delineation requires always both T2-weighted and volumetric T1-weighted sequences. T2-weighted fluid attenuated inversion recovery (FLAIR) sequences analyze the lesions surrounding brain tissues $[2,3]$.

Objective assessment of apparently healthy tissue surrounding brain tumors seems to be a considerable factor interfering not only with the radiosurgical procedure, but also with the recurrence rate and overall survival. Several studies identified infiltrative spectroscopic pattern of the perilesional edema in more than $96 \%$ of high-grade gliomas cases and in $11,5 \%$ of patients with brain metastasis [4]. Moreover, some autopsy series of brain metastases confirmed infiltrative growth in radiologically healthy surrounding tissues in more than $60 \%$ of cases. This unseen malignant component is responsible of $80 \%$ of "early recurrence" which should be considered as natural evolution of the main tumor [5]. In the management of high grade gliomas, the radiosurgeons are faced either to carcinologic incomplete procedures or to overestimated target irradiation with unbalanced benefit/risk action mostly related to radiation-induced brain necrosis [6]. The delineation of clinical target volume (CTV) which is defined as the volume of tissue that contains the GTV and any microscopic tumor or paths of spread, became a standard for any radio-surgical planning. Since a decade, the magnetic resonance spectroscopy (MRS) was standardized in the target volume assessment. The aim is to establish a metabolic lesional cartography. It had been reported that choline/ $\mathrm{N}$ - acetylaspartate (NAA) multivoxel MR spectroscopy index higher that 2,5 is in favor of malignancy in glioma with sensitivity of $90 \%$ and specificity of $85 \%$ [7]. However, NAA/Creatine $(\mathrm{Cr})$ and Choline/Cr ratios are more relevant in the analysis of perilesional edema in brain metastasis cases. The introduction of MRS metabolic cartography concept, the use of relevant metabolite and adapted metabolites ratio estimation contributed to precision in radiosurgery. However, MRS is not used for target delineation for Gamma Knife radiosurgical treatment because of its incompatibility with the Leksell Gamma Knife planning software. Recently, we described the development of the first software allowing the integration of metabolic cartography based on multivoxel spectroscopic MRI in the radiosurgical planning for Leksell Gamma Knife Radiosurgery.

The few existing meta-analysis could not lead to gold standard volume delineation techniques despite objective advance in imaging assessment [8,9]. Prospective studies using multimodal imaging data will help to overcome this insufficiency for target delineation in radiosurgery.

References

[1] Certo F, Altieri R, Maione M, Schonauer C, Sortino G, MD, Fiumanò G, et al. FLAIRectomy in supramarginal resection of Glioblastoma correlates with clinical outcome and survival analysis: A prospective, single institution case series. Op Neurosurg.2021 :20: 151-63. [2] Ajithkumar T, Horan G, Padovani L, Thorp N, Timmermann B, Alapetite C, et al. SIOPE - Brain tumor group consensus guideline on craniospinal target volume delineation for high-precision radiotherapy. Radiother Oncol. 2018; 128:192-97.

[3] Tong E, McCullagh KL, Iv M. Advanced Imaging of Brain Metastases: From Augmenting Visualization and Improving Diagnosis to Evaluating Treatment Response. Front Neurol. 2020;11:270.

[4] Lee $\mathrm{E}$, , Ahn K, Lee $\mathrm{EK}$, Lee $\mathrm{YS}$, Kim DB. Potental role of advanced MRI techniques for the peritumoural region in airferentiating glioblastoma multiforme and solitary metastatic lesions. Clin Radiol. 2013; 68 : e689-97.

5] Lee $C C$, Lee WK, W

[9] Akanda ZZ, Hong W, Nahavandi S, Haghighi N, Phillips C, Kok DL. Post-operative stereotactic radiosurgery following excision of brain metastases: A systematic review and meta-analysis. Radiother Oncol. 2020; 142:27-35.

Citation: Hassini Derkaoui F. Contribution of spectroscopic magnetic resonance imaging to target volume delineation in Gamma Knife Radiosurgery: Myth or reality? Jr.med.res. 2021; 4(1):2. Derkaoui Hassini@ All rights reserved.

https://doi.org/10.32512/jmr.4.1.2021/2

Submit your manuscript: www.jmedicalresearch.com 